\title{
El fin de la esperanza: los homicidios como causa de la expectativa de vida perdida
}

\section{The end of hope: homicides as cause of loss of life expectancy}

\author{
Manuel Ordorica-Mellado y Mauricio Pablo Cervantes-Salas
}

\author{
El Colegio de México, México \\ Centro de investigación en Ciencias de Información Geoespacial, \\ A.C. (CentroGeo)
}

\section{Resumen}

Este trabajo tiene por objetivo general, realizar un análisis de la dinámica de la violencia en México y sus entidades federativas, y su efecto en los niveles de la mortalidad. Las siguientes preguntas orientaron y dieron forma a la narrativa seguida en este documento: ¿Cómo se ha comportado la violencia en México a lo largo del siglo pasado y del siglo actual? ¿Es el clima de violencia referido un hecho inédito en estos años, o ha sido una constante en la historia mexicana? ¿Cómo ha cambiado este fenómeno en los territorios del país? ¿Cuál es el impacto de la violencia en los niveles de la mortalidad general? Los resultados muestran que el fenómeno de la violencia no es inédito, sin embargo, el número de muertes violentas sí, lo cual ha afectado de manera importante el incremento en la esperanza de vida al nacer, sobre todo entre los hombres, reflejo de una mayor tasa de homicidios en este grupo poblacional. Otro resultado importante del trabajo es que la esperanza de vida al nacer en México se ha mantenido sin cambios durante el siglo XXI, en alrededor de 75 años, hecho por el cual nuestro país pasará a la historia.

Palabras clave: Violencia, homicidios, esperanza de vida, México, entidades federativas.

Abstract

The main objective of this paper is analyze the dynamics of violence in Mexico and its federal entities, and the effect in mortality levels. The narrative was guided by the following questions: How has violence in Mexico developed over the past century and the current century? Is the climate of violence referred to as truly unprecedented, or has it been a constant in Mexican history? How has this phenomenon changed in the territories of the country? What is the impact of violence in population dynamics? One highlight is that the phenomenon of violence has always been present, however, the number of violent deaths is unprecedented in the history of Mexico. Another highlight, is that violent deaths has significantly affected the increase in life expectancy at birth, especially among men, reflecting a higher rate of homicides in this population group. An important result of this work is that life expectancy at birth has remained unchanged, in about 75 years, during the 21ST century. For this fact our country will go down in history because it has not increased its life expectancy at birth.

Keywords: Violence, homicides, life expectancy, Mexico, Federal entities. 


\title{
INTRODUCCIÓN
}

\author{
"La indiferencia del mexicano ante la muerte se nutre de su indi- \\ ferencia ante la vida. El mexicano no solamente postula la intrascen- \\ dencia de morir, sino la de vivir... Nuestra indiferencia ante la muerte \\ es la otra cara de nuestra indiferencia ante la vida. Matamos porque la \\ vida, la nuestra y la ajena, carece de valor". \\ Octavio Paz en: "Todos Santos, Día de Muertos". \\ El Laberinto de la Soledad, 1950.
}

A 1 adscribirse a la Declaración Universal de los derechos humanos, el Estado Mexicano tiene como principal responsabilidad dotar de las condiciones necesarias para asegurar el derecho a la vida y la seguridad de las personas. ${ }^{1} \mathrm{Al}$ respecto, Galtung refiere que en términos generales, la violencia puede ser definida como la privación de los derechos humanos fundamentales, como lo es el derecho a la vida, y distingue tres formas de Violencia: estructural, cultural y directa, que en su conjunto conforman un triángulo vicioso (Galtung, 2016: 150-54). En este trabajo concentramos la atención en el estudio de la violencia directa a través del análisis de las muertes por homicidio ${ }^{2}$ y su efecto en la esperanza de vida.

Algunos estudios señalan que en México el incremento de la violencia se ha sucedido como nunca antes, incluso calificando la situación como estado de guerra (Cendejas y Merino, 2016; Hiik, 2018). Al respecto, el Instituto Internacional de Estudios Estratégicos (IISS, 2017) señala que existe un conflicto armado entre el Estado Mexicano y los cárteles del narcotráfico. Tal situación es muestra del importante reto que enfrentan los gobiernos federal y estatal para la construcción de la paz y la seguridad ciudadana en los territorios del país.

Esto nos llevó a plantear las siguientes preguntas y objetivos de investigación: ¿Cómo se ha comportado la violencia en México a lo largo del siglo pasado y del siglo actual? ¿Es el clima de violencia referido realmente inédito, o ha sido una constante en la historia mexicana? ¿Cómo

\footnotetext{
1 Artículo 3 de la Declaración Universal de los Derechos Humanos.

2 Según el Génesis, el primer homicidio de la historia fue cuando Caín mató a Abel. Caín trabajaba ba de agricultor y Abel se dedicaba al pastoreo. Los dos presentaron sus sacrificios a Dios. Al verlos, Dios prefirió la ofrenda de Abel (de los primogénitos de sus ovejas) al de Caín (del fruto de la tierra). Caín enloqueció de celos y mató a su hermano. El asesinato de Abel se explica por la preferencia de Dios a los regalos del segundo hijo.

El homicidio es un delito que consiste en una acción mediante la cual se causa la muerte a otra persona ya sea dolosa o culposamente. El término procede del latín homicidium, de homo, "humano", y caedere "matar".

En el capítulo 22.15 del Apocalipsis se dice que el homicida no entrará en el Reino de Dios.
} 
ha cambiado este fenómeno en los territorios del país? ¿Cuál es el impacto de la violencia en la demografía? En concordancia con estas inquietudes, el objetivo general de este trabajo es realizar un análisis de la dinámica e impacto de la violencia en México y sus Entidades Federativas desde una perspectiva demográfica. Además, se analiza la estructura por edades de los homicidios. Una conclusión central del trabajo es que en lo que va del siglo XXI, la esperanza de vida al nacer se ha mantenido sin cambios en alrededor de 75 años, cuando hoy ya debería de haber llegado casi a los 79 años.

El artículo está conformado por seis apartados y las conclusiones. En el apartado uno se presenta una aproximación demográfica al análisis de la violencia; en el segundo, se analiza la evolución reciente en la esperanza de vida al nacimiento; en el tercer apartado se incluye la importancia de los homicidios como causa de muerte y su incidencia por edad y sexo en México; el cuarto apartado contiene una descripción de la violencia en México en el tiempo y en el quinto y último apartado se presenta el crecimiento en el número de homicidios por entidad federativa entre 2007 y 2018.

\section{Aproximación demográfiCa AL ANÁlisis de la VIOLENCia}

La violencia puede ser definida como la privación de los derechos humanos fundamentales, así como una disminución del nivel real de satisfacción de necesidades básicas por debajo de lo que es potencialmente posible (Galtung, 2016: 150-54). Galtung distingue tres formas de violencia; la estructural, la cultural y la directa, las cuales surgen cuando se ha fracasado en la transformación del conflicto (entendido como crisis y oportunidad, como objetivos incompatibles y relaciones de poderes; Calderón, 2009). La violencia estructural es "intrínseca a los sistema sociales, políticos y económicos mismos que gobiernan las sociedades, los estados y el mundo" (Calderón, 2009). Por su parte, la violencia cultural está referida a aquéllos "aspectos simbólicos de nuestra experiencia que pueden utilizarse para justificar o legitimar la violencia directa o estructural" (Ibidem). Finalmente, la violencia directa es la violencia manifiesta, la más evidente, y por lo general se manifiesta de manera física, verbal o psicológica (Ibidem). En este trabajo, centramos nuestra atención en el estudio de la violencia directa a través del análisis de las muertes por homicidio.

Conceptualmente, la definción de violencia directa de Galtung no es muy lejana a la definición de la Organización Mundial de la Salud (2003: 5), la cual define la violencia como "el uso intencional de la fuerza o el poder físico, de hecho o como amenaza, contra uno mismo, otra persona, o 
un grupo o comunidad, que cause o tenga mucha probabilidad de causar lesiones, muerte, daño psicológico, trastornos del desarrollo o privaciones".

De acuerdo con las características de los que cometen el acto de violencia, la Organización Mundial de la Salud (2003: 6) reconoce tres tipos de expresión de la violencia. La violencia autoinflingida, se refiere a la violencia que una persona ejerce sobre sí misma. La violencia interpersonal es aquélla que es impuesta por otro individuo o un número pequeño de individuos. Finalmente, la violencia colectiva es infligida por grupos grandes, como puede ser el Estado, contingentes políticos organizados y organizaciones terroristas, entre otras. No obstante, los observadores internacionales concuerdan en señalar que el incremento de las muertes violentas en México, principalmente se deben a los dos últimos tipos de violencia, lo cual se explica por la confrontación (o conflicto no resuelto, si se retoma a Galtung, 2016) entre el Estado Mexicano y el narcotráfico (Hiik, 2018).

Entre las diversas formas que propone Galtung en que se puede expresar la violencia directa (mutilaciones, acoso, sanciones, miseria, des-socialización, resocialización, represión, detención expulsión, entre otros; Calderón, 2009), en este trabajo analizamos la muerte. La mortalidad ocurrida dentro de una población en un periodo dado de tiempo, junto con la fecundidad y la migración, es uno de los tres componentes del cambio demográfico. Si se asocia a los homicidios, entendidos como las muertes por agresión con independencia de su calificación jurídica, la mortalidad puede ser empleada como un indicador universal de la violencia (Chaparro-Narváez, Cotes-Cantillo, León-Quevedo, Castañeda-Orjuela, 2016).

Cuando se presenta una elevada incidencia de homicidios, pudiera haber un efecto importante en la disminución de la esperanza de vida al nacimiento, tal es el caso de México. En 2017 se presentaron 703,047 defunciones de las cuales 32,079 fueron homicidios, lo que representa 4.6 por ciento de las muertes totales. Si le aplicamos 4.6 por ciento a la esperanza de vida al nacer de 75 años, obtenemos que México ha perdido aproximadamente 3.5 años en su esperanza de vida al nacer.

Así, en este trabajo las muertes por homicidios y su efecto en la esperanza de vida, son entendidos como dos indicadores que dan cuenta de la magnitud en la privación del derecho humano a la vida, y por tanto, como indicadores de la violencia directa.

En una población estacionaria, la esperanza de vida al nacer es igual al inverso de la tasa de mortalidad. En una población estacionaria la tasa de crecimiento demográfico es cero y su estructura por edad permanece sin 
cambios en el tiempo (Lotka, 1969). En el caso de las poblaciones estables la estructura por edad está dada por:

$$
c(a)=b e^{-r a} p(a)
$$

Donde c(a) es el número de individuos a la edad $a$ (coeficiente de distribución por edad); $b$ es la tasa de natalidad; $r$ es la tasa de crecimiento y $\mathrm{p}(\mathrm{a})$ es la probabilidad de que una persona de edad 0 llegue con vida a la edad $a$.

Si se asume que todos los individuos de la población están distribuidos entre las edades 0 e $\infty$, y que el total de individuos representan la unidad, al integrar los dos lados de la ecuación, se tiene:

$$
1=b \int_{0}^{\infty} e^{-r a} \mathrm{p}(\mathrm{a}) \mathrm{da}
$$

Si $r=0$ y por tanto $b=d$, que es el caso de una población estacionaria, que se supone cerrada a la migración, la ecuación se transforma en lo siguiente:

$$
1=b \int_{0}^{\infty} p(a) d a
$$

En esa ecuación, $\int_{0}^{\infty} p(a) d a$ es igual a la esperanza de vida al nacer $\left(\mathrm{e}_{0}^{0}\right)$, por lo que se tiene:

$$
1=b e_{0}^{0}
$$

Como $b=d$, se tiene también:

$$
1=d e_{0}^{0}
$$

Esto significa que la esperanza de vida al nacer es igual al inverso de la tasa bruta de mortalidad en una población estacionaria.

$$
e_{0}^{0}=\frac{1}{d}
$$

Si aplicamos el porcentaje de homicidios de 4.6 por ciento a la esperanza de vida al nacimiento obtenemos una aproximación de lo que México ha perdido en su esperanza de vida al nacer. México no ha llegado a una población estacionaria porque su tasa de crecimiento demográfico es distinta de cero y su estructura por edad presenta cambios en el tiempo. 
Esta relación se constata también con la siguiente ecuación:

$$
\begin{aligned}
e_{x}^{0} & =\frac{1}{l(x)} \int_{0}^{\infty} l(x+t) d t \\
& =\int_{0}^{\infty} e^{-\int_{0}^{t} \mu(x+w) d w} d t
\end{aligned}
$$

Si $\mu$ es constante se tiene que:

$$
=\int_{0}^{\infty} e^{-\mu t} d t=\frac{1}{\mu}
$$

En particular, cuando $x=0$ el inverso de la tasa instantánea de mortalidad es igual a la esperanza de vida al nacer:

$$
e_{0}^{0}=\frac{1}{\mu}
$$

Donde $e_{x}^{0}$ es la esperanza de vida a la edad $x, l(x)$ es el número de sobrevivientes a la edad $x, t$ es el tiempo y $\mu$ es la tasa instantánea de mortalidad.

\section{LA EVOLUCIÓN RECIENTE EN LA ESPERANZA DE VIDA}

\section{AL NACIMIENTo EN MÉXICO}

A lo largo de casi un siglo, es decir de 1921 a 2017, la esperanza de vida al nacer pasó de 32.6 años a 74.9 años; 42.3 años de incremento (Bravo Becherelle y Reyes Jiménez, 1958). Tres quintas partes de ese incremento ocurrieron en poco más de medio siglo, entre 1950 y 2017, periodo durante el cual la esperanza de vida al nacimiento se incrementó en 26.2 años, al pasar de 48.7 años (Camposortega, 1992) a los 74.9 años ya mencionados (CONAPO, 2017) (Ver Tabla 1). Este cambio ha sido uno de los más grandes logros en nuestro país, y el sector salud uno de los grandes actores en estos éxitos. Cumplió con sus propósitos al bajar los niveles de fecundidad con un exitoso programa de planificación familiar y salud reproductiva entre 1977 y el año 2000 que, en conjunto con acciones de comunicación y educación en población, permitieron reducir los niveles de fecundidad, con lo que la tasa de crecimiento demográfico llegó a uno por ciento anual en 2019. Según CONAPO, en 2019 alcanzamos una tasa global de fecundidad 
de 2.08, cifra que ubica a las poblaciones en el nivel de reemplazo. Ya se cumplió la meta en la tasa de crecimiento demográfico planteada en 1977 para el fin del siglo XX. Según las proyecciones de población de CONAPO publicadas en 2018, México en 2019 alcanzó ya la tasa meta de crecimiento total de la población de 1.01 por ciento (CONAPO, 2017). ${ }^{3}$ Recordemos que México en 1977 planteó una meta de crecimiento demográfico de uno por ciento para el año 2000.

Tabla 1: Estados Unidos Mexicanos: Esperanza de vida total, hombres y mujeres, 1990-2017

\begin{tabular}{lccc}
\hline Año & Total & Hombres & Mujeres \\
\hline $1990^{*}$ & 70.4 & 67.0 & 74.0 \\
$1995^{*}$ & 72.2 & 69.2 & 75.3 \\
$2000^{*}$ & 73.2 & 70.5 & 76.1 \\
$2005^{*}$ & 73.9 & 71.3 & 76.7 \\
$2010^{*}$ & 74.0 & 71.0 & 76.9 \\
$2015^{* *}$ & 74.7 & 71.8 & 77.6 \\
$2016^{* *}$ & 74.8 & 72.0 & 77.7 \\
$2017^{* *}$ & 74.9 & 72.0 & 77.8 \\
\hline
\end{tabular}

* Fuente: Consejo Nacional de Población (CONAPO). Estimaciones y proyecciones de la población por entidad federativa, 2010-2050.

** Consejo Nacional de Población (CONAPO). Proyecciones de la población de México y de las entidades federativas, 2016-2050, publicado en 2018-09.

No obstante esos logros, entre 2006 y 2015 ocurrió un hecho insólito, sólo visto en momentos de guerra: el incremento en la esperanza de vida al nacer se frenó súbitamente. Uno podría pensar que ello se debe a que en México llegamos a un límite biológico en el número medio de años de vida desde el nacimiento y a los rezagos en el sector salud. El freno en la esperanza de vida no es atribuible a las muertes por enfermedad, y el comportamiento en la esperanza de vida al nacimiento en otros países indica que tampoco se explica por un límite biológico. Este freno en la esperanza de vida se explica mejor por el incremento acelerado de las muertes por homicidio en México.

Al inicio del siglo XXI teníamos una visión optimista en la evolución de la esperanza de vida al nacer. Cuando en 2002 el Consejo Nacional de

\footnotetext{
${ }^{3}$ Aunque se llegó con 19 años de retraso al nivel de crecimiento demográfico programado, y con la ayuda de la emigración internacional, dejaron de nacer alrededor de 50 millones de personas. Con base en las proyecciones de población elaboradas a partir del censo del año 2000, para 2015 ya deberíamos de estar por debajo del nivel reemplazo, en 1.91 hijos (CONAPO, 2002) en promedio.
} 
Población publicó las proyecciones de la población de México, la esperanza de vida al nacer proyectada al 2015 fue de 77.6 años (75.2 para los hombres y 80.0 para las mujeres, CONAPO, 2002) y sólo llegamos a 74.7 años (CONAPO, 2018). Esto significa que en este periodo perdimos 2.9 años en la esperanza de vida al nacer.

Un hecho que ejemplifica la erosión de la esperanza de vida por violencia se observa cuando analizamos Ciudad Juárez, en esta ciudad la esperanza de vida al nacer de las mujeres no sólo no aumentó sino que se redujo de 72.9 en 2000 a 72.1 años en 2010, lo cual es un hecho insólito. Algo todavía más grave ocurrió en Ciudad Juárez en ese mismo periodo con los hombres; la esperanza se redujo de 66.8 años en 2000 a 57.7 años en 2010, perdiendo 9.1 años en el número medio de años que se espera viva una persona desde su nacimiento (Ordorica, 2015). Esto ocurre en varios estados del país donde la violencia es muy elevada, pero éste es uno de los casos más alarmantes del país.

Salama (2013: 15), reporta que los hechos de violencia afectan de manera importante la esperanza de vida, sobre todo la masculina, y ofrece los siguientes ejemplos para Brasil: en la ciudad de Recife la esperanza de vida al nacer en 2000 para los hombres disminuyó 2.89 años, 2.21 años en Río de Janeiro y 2.48 años en Sao Paulo, reducciones que, según el autor, estuvieron directamente relacionadas con el incremento en las tasas de homicidios.

Japón en contraparte, es un ejemplo a nivel mundial respecto al incremento en la esperanza de vida, y la comparación con este país es pertinente porque es la nación con la esperanza de vida al nacer más elevada del mundo. En 1975-1980 Japón tenía una esperanza de vida al nacer de 75.4 años y ya para el periodo 2010-2015 su esperanza de vida era de 83.3 años (United Nations, 2017). En 35 años, Japón aumentó su esperanza de vida en 7.9 años. En 1990-1995 Japón llegó a 79.4 años, un aumento de cuatro años en su esperanza de vida al nacer en 15 años (United Nations, 2017). $\mathrm{Si}$ en México la esperanza de vida hubiera seguido el incremento experimentado en Japón una vez llegado a 75 años de esperanza de vida, en 2015 deberíamos de haber alcanzado los 79 años en la esperanza de vida al nacer. Bajo este supuesto, México ya perdió casi cuatro años en la esperanza de vida y la alta incidencia de homicidios a edades jóvenes puede constatar dicho comportamiento. ${ }^{4}$

4 United Nations, Departament of Economic and Social Affairs, Population Division (2017). World Population Prospects. The 2017 Revision. 


\section{LA IMPORTANCIA DE LOS HOMICIDIOS COMO CAUSA DE MUERTE Y SU INCIDENCIA POR EDAD Y SEXO EN MÉXICO}

En México las muertes violentas siempre han sido mucho más elevadas en los hombres que, en las mujeres, y adquieren mayor importancia en edades jóvenes. Al respecto, Camposortega (1989: 245) señala que en 1980 las muertes violentas eran la principal causa de muerte entre los hombres a partir de los cinco años, y esas muertes explicaban entre 45 y 58 por ciento del total de las muertes ocurridas de los cinco a los 40 años. El mismo autor señala que las ganancias anuales en la esperanza de vida pasan de 0.9 años durante los años cincuenta a 0.3 años durante los años sesenta, lo cual se encuentra asociado al incremento de las muertes por accidentes y violencia, así como a enfermedades circulatorias y de cáncer (Camposoratega, 1989: 251). Por su parte, entre 1970 y 1980 las mujeres lograron ganancias anuales en su esperanza de vida en el orden de los 0.6 años, mientras que los hombres de 0.4 años, diferencia que se explica por el incremento de las muertes violentas entre los hombres adultos (Camposoratega, 1989: 251). En 1992, las muertes por homicidios ocupaban el primer lugar entre las causas de defunción de los varones en edades productivas (Victoria, Hijar, Rascón y Blanco, 1996).

Los homicidios ocupan cada vez más un lugar muy importante dentro de las causas de muerte en México. Para los años 2016 y 2017, los homicidios ocuparon el séptimo lugar entre las primeras 20 causas de muerte en el país. Antes que esta causa de muerte, en el primer lugar están las enfermedades del corazón; en segundo lugar, la diabetes mellitus; en tercer lugar, los tumores malignos; en cuarto lugar, las enfermedades del hígado, y el quinto lugar lo ocupan los accidentes (Tabla 2).

La actual distribución por sexo de la población permite decir que México está a tiempo de poder aprovechar lo que se ha dado en llamar el bono demográfico. Una manera sencilla de definir el bono o dividendo demográfico es con el índice de dependencia. ${ }^{5}$ La relación de dependencia se calcula para 2018 en 48 personas en edades no activas por 100 personas en edades activas, según la información que produce Naciones Unidas en su revisión de 2017 (United Nations, 2017).

Nuestro valor máximo se ubicó en el año de 1970 en 101 personas en edades no activas por 100 en edades activas.

\footnotetext{
5 El índice de dependencia es la relación que hay entre la suma de la población de 0 a 14 años y la de 65 años o más, que son las personas en edades no activas, entre la población de 15 a 64 años, que se consideran las personas en edades activas.
} 
Tabla 2: Las principales causas de muerte en México, 2016 y 2017

\begin{tabular}{|c|c|c|}
\hline Año & 2016 & 2017 \\
\hline Total & 685,766 & 703,047 \\
\hline Enfermedades del corazón & 136,342 & 141,619 \\
\hline Diabetes mellitus & 105,572 & 106,525 \\
\hline Tumores malignos & 82,502 & 84,142 \\
\hline Enfermedades del hígado & 38,755 & 38,833 \\
\hline Accidentes & 37,418 & 36,215 \\
\hline Enfermedades cerebrovasculares & 34,782 & 35,248 \\
\hline Agresiones & 24,559 & 32,079 \\
\hline Enfermedades pulmonares obstructivas crónicas & 23,271 & 22,954 \\
\hline Influenza y neumonía & 21,572 & 21,892 \\
\hline Insuficiencia renal & 13,132 & 13,167 \\
\hline Ciertas afecciones originada en el periodo perinatal & 12,641 & 13,060 \\
\hline $\begin{array}{l}\text { Malformaciones congénitas, deformidades y anomalías } \\
\text { cromosómicas }\end{array}$ & 9,157 & 9,259 \\
\hline Desnutrición y otras deficiencias nutricionales & 7,388 & 7,163 \\
\hline Lesiones autoinflingidas intencionalmente & 6,370 & 6,559 \\
\hline Bronquitis crónica y la no especificada, enfisema y asma & 5,280 & 4,970 \\
\hline Enfermedad por virus de la inmunodeficiencia humana & 4,630 & 4,720 \\
\hline Enfermedades infecciosas intestinales & 3,908 & 3,621 \\
\hline Anemias & 3,504 & 3,422 \\
\hline Septicemia & 2,744 & 2,856 \\
\hline Úlceras gástrica y duodenal & 2,720 & 2,716 \\
\hline Subtotal & 576,247 & 591,020 \\
\hline $\begin{array}{l}\text { Síntomas, signos y hallazgos anormales clínicos } \\
\text { y de laboratorio, no clasificados en otra parte }\end{array}$ & 9,780 & 9,943 \\
\hline Las demás causas & 99,739 & 102,084 \\
\hline
\end{tabular}

Fuente: INEGI. Consulta interactiva de datos. Defunciones generales, causas detalladas CIE y Lista Mexicana de Enfermedades.

El valor mínimo se encontrará en 12 años más cuando sea de 47.4 personas en edades no activas por 100 en edades activas. Alrededor de 2030 estaremos en el mejor momento respecto al dividendo demográfico. Esto quiere decir que todavía tenemos poco más de una década para aprovechar nuestro bono demográfico (ver Figura 1). 
Figura 1: Población de 0 a 14 años y 65 o más en relación con las de 15 a 64 años

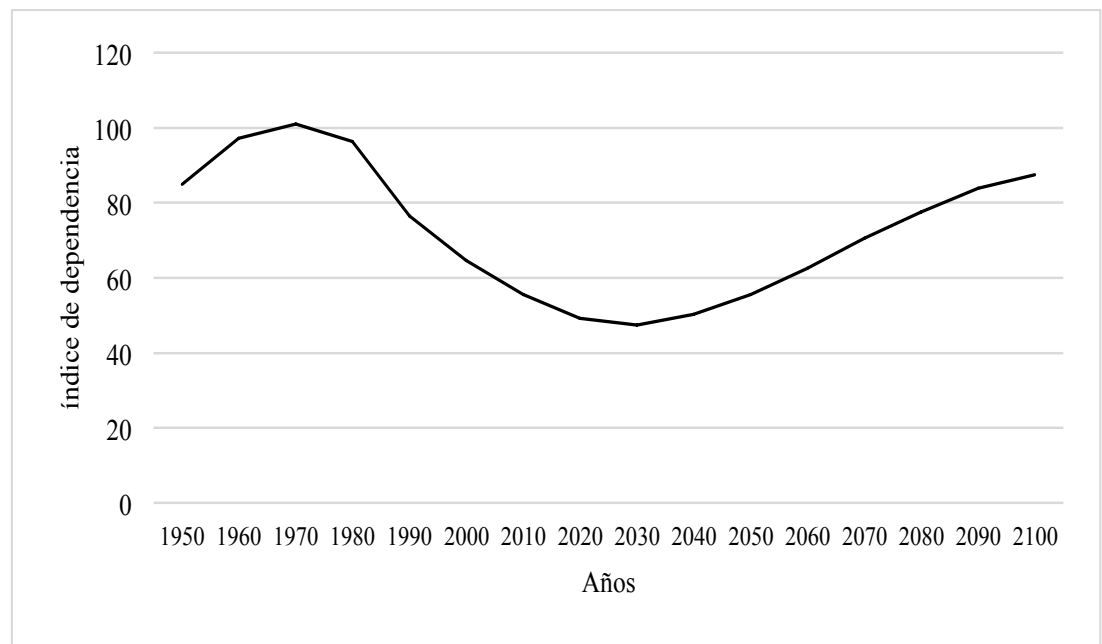

Fuente: United Nations. Population Division (2017). World Population Prospects: The 2017 Revision.

La importancia del bono demográfico radica en conocer y contrastar el monto de población que está en edad de aportar con su trabajo, los bienes $\mathrm{y}$ recursos económicos que el país necesita, respecto al monto poblacional que por su edad no está en condiciones de producir esos bienes y recursos.

Si bien una buena parte de la población actual se encuentra en edades productivas, las condiciones de violencia que existen en el país impiden aprovechar ese bono demográfico porque muchos de esos jóvenes fallecen asesinados, perdiendo así un capital humano que se encuentra en la plenitud del desarrollo de sus potencialidades.

Cuando se analiza la estructura por edad de los homicidios (Figura 2), se aprecia que entre los 0 y hasta los 14 años la incidencia de este fenómeno es baja, pero al pasar al siguiente grupo de edades, el de 15 a 19 años, la cifra de homicidios crece aceleradamente hasta alcanzar su máximo entre los 25 y 29 años, para reducirse paulatinamente a partir de los 30-34 años.

$\mathrm{Al}$ igual que en otros momentos de la historia de México, las muertes por homicidios son un fenómeno que se sucede principalmente entre los varones (ver Tabla 3). En 2017 hubo 8.3 veces más muertes de hombres que de mujeres. Esa razón empieza a crecer aceleradamente a partir de los 15-19 años y tiene su máximo en los 40-44 años, siendo 9.3 veces mayor el número de homicidios de varones respecto al número de mujeres. De los 20 a los 70 años la relación de homicidios supera el valor de las mujeres 
en más de siete veces, y se reduce a una razón por debajo de cuatro veces a partir de los 75 años.

Figura 2: E.U.M. Homicidios por grupos de edades y sexo, 2017

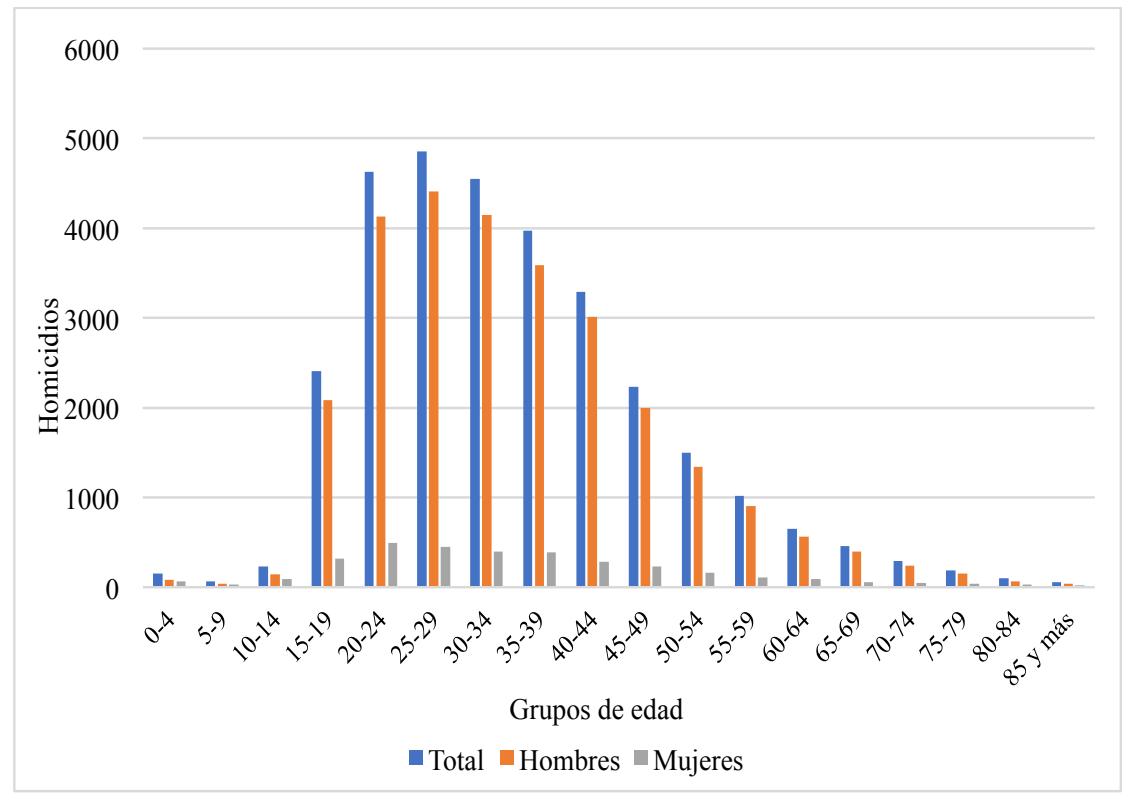

Fuente: United Nations. Population Division (2017). World Population Prospects: The 2017 Revision.

Como se aprecia en la Figura 2, un importante número de las muertes por homicidios en el país ocurren en edades jóvenes y productivas, lo que en gran parte origina el estancamiento en la esperanza de vida al nacer.

\section{Las MUertes POR AGRESIONES EN MÉXICO EN EL TIEMPO}

Las tasas de mortalidad por homicidios nos permiten conocer qué tan generalizado es el fenómeno de la violencia en una población, en tal sentido, este fenómeno tiende a "diluirse" mientras más personas formen parte de dicha población; a niveles constantes de homicidios y a mayor número de individuos en una población, menor será el valor que adopte la tasa.

Por su parte, es importante destacar que la inscripción tardía y el subregistro de la información contenida en los registros administrativos, generan fluctuaciones en el comportamiento anual de los datos, por ello, en este trabajo se asumen que las variaciones en el comportamiento de los homicidios es el mismo en todos los años. Si bien los datos podrían ser suavizados 
utilizando diversas estrategias de graduación de carácter actuarial (véase Silva et al., 2014), en este trabajo hemos decidido no recurrir a estos métodos, porque ello impediría observar cambios que pueden ser importantes para los objetivos de análisis de este trabajo.

Tabla 3: E.U.M. Homicidios por grupos de edades y sexo, 2017

\begin{tabular}{|c|c|c|c|c|c|}
\hline Edad & Total & Hombres & Mujeres & No esp. & $\begin{array}{l}\text { Razón de homicidios de } \\
\text { hombres respecto al número } \\
\text { de homicidios de mujeres }\end{array}$ \\
\hline Total & 32,079 & 28,522 & 3,430 & 127 & 8.3 \\
\hline $0-4$ & 152 & 86 & 65 & 1 & 1.3 \\
\hline $5-9$ & 69 & 40 & 29 & & 1.4 \\
\hline $10-14$ & 234 & 147 & 87 & & 1.7 \\
\hline $15-19$ & 2,403 & 2,083 & 320 & & 6.5 \\
\hline $20-24$ & 4,626 & 4,129 & 496 & 1 & 8.3 \\
\hline $25-29$ & 4,857 & 4,406 & 450 & 1 & 9.8 \\
\hline $30-34$ & 4,548 & 4,148 & 397 & 3 & 10.4 \\
\hline $35-39$ & 3,972 & 3,583 & 386 & 3 & 9.3 \\
\hline $40-44$ & 3,293 & 3,009 & 282 & 2 & 10.7 \\
\hline $45-49$ & 2,233 & 1,998 & 235 & & 8.5 \\
\hline $50-54$ & 1,499 & 1,338 & 161 & & 8.3 \\
\hline $55-59$ & 1,019 & 908 & 111 & & 8.2 \\
\hline $60-64$ & 646 & 559 & 87 & & 6.4 \\
\hline $65-69$ & 455 & 395 & 59 & 1 & 6.7 \\
\hline $70-74$ & 294 & 243 & 51 & & 4.8 \\
\hline $75-79$ & 189 & 150 & 39 & & 3.8 \\
\hline $80-84$ & 99 & 66 & 33 & & 2.0 \\
\hline 85 o más & 58 & 38 & 20 & & 1.9 \\
\hline No esp. & 1,433 & 1,196 & 122 & 115 & 9.8 \\
\hline
\end{tabular}

Fuente: INEGI.

No esp. (No especificados).

Pese a su arbitrariedad, se considera que una tasa de homicidios que llegue a 100 por cada cien mil habitantes, es un umbral a partir del cual la violencia se vuelve incontrolable (Salama, 2013: 12).

Teniendo en cuenta el umbral mencionado, en la Tabla 4 se puede apreciar que México ha mostrado ser un país muy violento a lo largo de su 
historia. Durante el periodo de tiempo que va de 1931 hasta el año 2017, la tasa de homicidios más alta se registró en 1940, alcanzando 67.04 homicidios por cada 100 mil habitantes, mientras que la más baja se observó en 2007 con 8.24 homicidios por cien mil habitantes.

Tabla 4: Estados Unidos Mexicanos: Número de homicidios, Población y tasa de defunciones por homicidio (por cien mil), 1931-2017

\begin{tabular}{|c|c|c|c|}
\hline Años & Homicidios & Población & Tasa de homicidios* \\
\hline 1931 & 8,551 & $16,839,399$ & 50.78 \\
\hline 1932 & 9,983 & $17,131,041$ & 58.27 \\
\hline 1933 & 7,904 & $17,427,734$ & 45.35 \\
\hline 1934 & 7,232 & $17,729,565$ & 40.79 \\
\hline 1935 & 8,098 & $18,036,623$ & 44.90 \\
\hline 1936 & 11,317 & $18,349,000$ & 61.68 \\
\hline 1937 & 11,266 & $18,666,787$ & 60.35 \\
\hline 1938 & 11,468 & $18,990,077$ & 60.39 \\
\hline 1939 & 11,445 & $19,318,966$ & 59.24 \\
\hline 1940 & 13,175 & $19,653,552$ & 67.04 \\
\hline 1941 & 10,434 & $20,194,997$ & 51.67 \\
\hline 1942 & 9,459 & $20,751,359$ & 45.58 \\
\hline 1943 & 9,809 & $21,323,048$ & 46.00 \\
\hline 1944 & 9,896 & $21,910,486$ & 45.17 \\
\hline 1945 & 10,815 & $22,514,109$ & 48.04 \\
\hline 1946 & 11,592 & $23,134,361$ & 50.11 \\
\hline 1947 & 12,124 & $23,771,700$ & 51.00 \\
\hline 1948 & 12,438 & $24,426,598$ & 50.92 \\
\hline 1949 & 12,404 & $25,099,538$ & 49.42 \\
\hline 1950 & 12,403 & $25,791,017$ & 48.09 \\
\hline 1951 & 11,889 & $26,584,772$ & 44.72 \\
\hline 1952 & 11,901 & $27,402,955$ & 43.43 \\
\hline 1953 & 10,805 & $28,246,320$ & 38.25 \\
\hline 1954 & 10,954 & $29,115,640$ & 37.62 \\
\hline 1955 & 10,782 & $30,011,715$ & 35.93 \\
\hline 1956 & 10,543 & $30,935,367$ & 34.08 \\
\hline 1957 & 10,446 & $31,887,447$ & 32.76 \\
\hline 1958 & 10,230 & $32,868,827$ & 31.12 \\
\hline 1959 & 11,250 & $33,880,412$ & 33.21 \\
\hline 1960 & 11,158 & $34,923,129$ & 31.95 \\
\hline
\end{tabular}


El fin de la esperanza: los homicidios como causa de la expectativa de vida perdida / M. ORDORICA y M.P. CERVANTES

Tabla 4: Continuación

\begin{tabular}{|c|c|c|c|}
\hline Años & Homicidios & Población & Tasa de homicidios* \\
\hline 1961 & 10,602 & $36,068,599$ & 29.39 \\
\hline 1962 & 9,493 & $37,251,640$ & 25.48 \\
\hline 1963 & 8,438 & $38,473,484$ & 21.93 \\
\hline 1964 & 8,781 & $39,735,405$ & 22.10 \\
\hline 1965 & 8,231 & $41,038,716$ & 20.06 \\
\hline 1966 & 8,234 & $42,384,775$ & 19.43 \\
\hline 1967 & 8,632 & $43,774,985$ & 19.72 \\
\hline 1968 & 8,711 & $45,210,794$ & 19.27 \\
\hline 1969 & 4,625 & $46,693,697$ & 9.90 \\
\hline 1970 & 8,450 & $48,225,238$ & 17.52 \\
\hline 1971 & 7,808 & $49,825,885$ & 15.67 \\
\hline 1972 & 7,505 & $51,479,660$ & 14.58 \\
\hline 1973 & 7,059 & $53,188,325$ & 13.27 \\
\hline 1974 & No disp.. & $54,953,703$ & 13.70 \\
\hline 1975 & No disp.. & $56,777,675$ & 17.70 \\
\hline 1976 & No disp.. & $58,662,188$ & 16.50 \\
\hline 1977 & No disp.. & $60,609,249$ & 19.00 \\
\hline 1978 & No disp.. & $62,620,935$ & 18.00 \\
\hline 1979 & 11,852 & $64,699,391$ & 18.32 \\
\hline 1980 & 12,225 & $66,846,833$ & 18.29 \\
\hline 1981 & 12,596 & $68,163,974$ & 18.48 \\
\hline 1982 & 13,323 & $69,507,067$ & 19.17 \\
\hline 1983 & 12,918 & $70,876,625$ & 18.23 \\
\hline 1984 & 12,473 & $72,273,168$ & 17.26 \\
\hline 1985 & 14,961 & $73,697,228$ & 20.30 \\
\hline 1986 & 15,909 & $75,149,348$ & 21.17 \\
\hline 1987 & 15,722 & $76,630,080$ & 20.52 \\
\hline 1988 & 15,204 & $78,139,989$ & 19.46 \\
\hline 1989 & 15,399 & $79,679,648$ & 19.33 \\
\hline 1990 & 14,497 & $81,249,645$ & 17.84 \\
\hline 1991 & 15,129 & $82,743,216$ & 18.28 \\
\hline 1992 & 16,596 & $84,264,243$ & 19.70 \\
\hline 1993 & 16,044 & $85,813,230$ & 18.70 \\
\hline 1994 & 15,840 & $87,390,691$ & 18.13 \\
\hline 1995 & 15,616 & $88,997,149$ & 17.55 \\
\hline
\end{tabular}


Tabla 4: Continuación

\begin{tabular}{|c|c|c|c|}
\hline Años & Homicidios & Población & Tasa de homicidios* \\
\hline 1996 & 14,508 & $90,633,139$ & 16.01 \\
\hline 1997 & 13,558 & $92,299,202$ & 14.69 \\
\hline 1998 & 13,658 & $93,995,892$ & 14.53 \\
\hline 1999 & 12,249 & $95,723,770$ & 12.80 \\
\hline 2000 & 10,743 & $97,483,412$ & 11.02 \\
\hline 2001 & 10,286 & $98,874,528$ & 10.40 \\
\hline 2002 & 10,089 & $100,285,496$ & 10.06 \\
\hline 2003 & 10,088 & $101,716,599$ & 9.92 \\
\hline 2004 & 9,330 & $103,168,124$ & 9.04 \\
\hline 2005 & 9,926 & $104,640,363$ & 9.49 \\
\hline 2006 & 10,452 & $106,133,611$ & 9.85 \\
\hline 2007 & 8,867 & $107,648,168$ & 8.24 \\
\hline 2008 & 14,006 & $109,184,339$ & 12.83 \\
\hline 2009 & 19,803 & $110,742,431$ & 17.88 \\
\hline 2010 & 25,757 & $112,322,757$ & 22.93 \\
\hline 2011 & 27,213 & $113,940,000$ & 23.88 \\
\hline 2012 & 25,967 & $115,570,000$ & 22.47 \\
\hline 2013 & 23,063 & $117,210,000$ & 19.68 \\
\hline 2014 & 20,010 & $118,056,000$ & 16.95 \\
\hline 2015 & 20,762 & $119,530,753$ & 17.37 \\
\hline 2016 & 23,953 & $121,023,966$ & 19.79 \\
\hline 2017 & 32,079 & $124,041,731$ & 25.86 \\
\hline $2018^{*}$ & 35,964 & - & 26.00 \\
\hline
\end{tabular}

Fuente: Instituto Nacional de Estadística y Geografía (INEGI); Sistema Nacional de Información de Salud (SINAIS); Panamerican Health Organization (PAHO).

México, tasas de homicidios por 100 mil habitantes desde 1931 a 2016. Recopilación del Ingeniero Manuel Aguirre Botello con datos de INEGI, SINAIS, PAHO, Presidencia y Lantia.

Para 2017, estimaciones propias a partir de las Estadísticas Vitales y de las Proyecciones de Población de México y de las entidades federativas, 2016-2050, publicadas por CONAPO en 2018.

*Para 2018 el INEGI publicó las cifras preliminares con corte al 15 de julio de 2019. Estadísticas Vitales, Defunciones Registradas.

De 1931 a 1952, es decir, durante 21 años, las tasas de mortalidad por homicidio fueron especialmente altas, y oscilaron entre las cuarenta y las casi setenta defunciones por cada cien mil habitantes. A partir de 1953 se presenta un descenso continuo hasta el año de 1973, periodo en el que las tasas de homicidios se redujeron de 38.25 a 13.27 por cien mil habitantes. A partir de 1974 inicia un nuevo periodo de 13 años en el que se incremen- 
tan las tasas de homicidios, pasando de 13.27 a 20.52 por cien mil habitantes en 1987. A partir de 1988 los homicidios se reducen hasta alcanzar niveles especialmente bajos y nunca antes vistos en la historia de México. En 1988 la tasa de homicidios era de 19.46 por cien mil, para reducirse hasta 8.24 homicidios por cien mil en 2007. Finalmente, a partir del 2008 y hasta el 2017 las tasas de homicidios se vuelven a incrementar, oscilando entre 12.83 y 25.86 homicidios por cien mil personas (Véase Tabla 4 y figuras 3 y 4 ).

Si bien en 2017 la tasa de mortalidad por homicidios era aproximadamente la mitad de la registrada en 1931 (25.86 vs 50.78 por cien mil, respectivamente), las actuales tasas no son para sentir consuelo alguno, porque son cifras elevadas y están en aumento. Entre 1982 y 2015 han muerto por homicidios alrededor de 520 mil personas. Esta cifra es equivalente a la población de la ciudad de Morelia, la cual alcanzaba, según el censo, un total de 597,511 habitantes en 2010, pudiendo imaginar así, un panteón con las mismas dimensiones que esa ciudad con personas enterradas por la violencia. Destaca que en 2016 y 2017, ocurrieron un total de 56,032 homicidios, es decir, los homicidios ocurridos en tan solo dos años, representan 10.7 por ciento de los ocurridos entre 1982 y el 2017. De seguir así, en un periodo de 20 años, ocurrirían los homicidios que se sucedieron en un periodo de 33 años (1982-2017).

En 2017 el número de personas muertas por homicidio fue de 32,079; 28,522 hombres y 3,430 mujeres, más 127 no especificados. ${ }^{6}$ El 2017 será uno de los años en que ocurrieron más homicidios en la historia de nuestro país, de los cuales se sucedieron 8.4 homicidios de hombres por cada homicidio de una mujer. Los 32,079 homicidios ocurridos en 2017, en promedio equivalen a poco más de dos mil quinientos homicidios por mes y a casi 90 asesinatos diarios. Tal parece que las acciones contra el narco a partir de 2007 tuvieron un efecto perverso y negativo.

Veamos a continuación, cómo ha cambiado el comportamiento de la violencia en las entidades federativas del país.

${ }^{6}$ INEGI, Estadísticas Vitales. 
Figura 3: E.U.M. Número de homicidios, 1982-2017

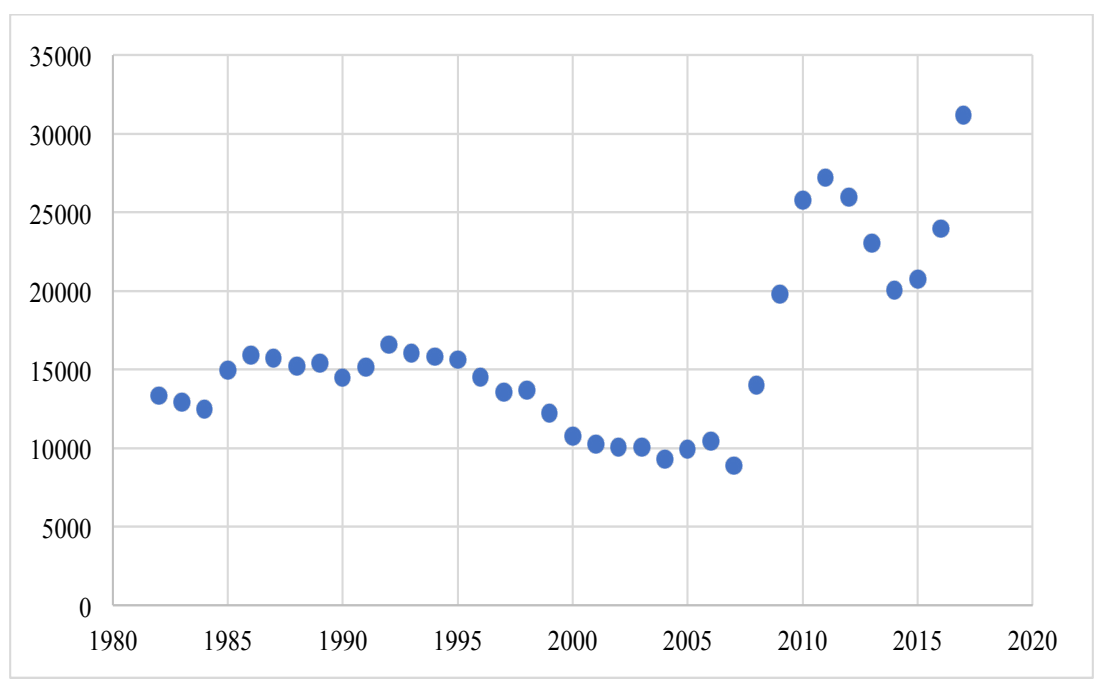

Fuente: Instituto Nacional de Estadística y Geografia (INEGI); Sistema Nacional de Información de Salud (SINAIS); Panamerican Health Organization (PAHO).

Figura 4: E.U.M. Homicidios por cada cien mil habitantes, 1931-2017

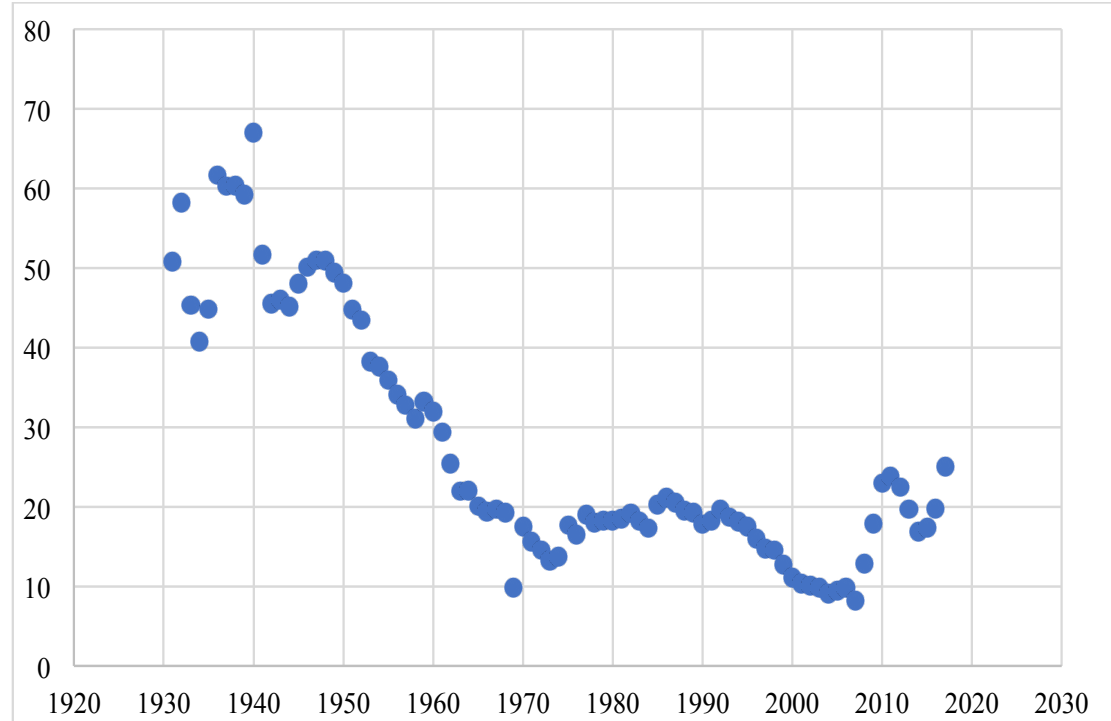

Fuente: Instituto Nacional de Estadística y Geografía (INEGI); Sistema Nacional de Información de Salud (SINAIS); Panamerican Health Organization (PAHO).

México, tasas de homicidios por 100 mil habitantes desde 1931 a 2016. Recopilación del Ingeniero Manuel Aguirre Botello, con datos de INEGI, SINAIS, PAHO, Presidencia y Lantia. 
El fin de la esperanza: los homicidios como causa de la expectativa de vida perdida / M. ORDORICA y M.P. CERVANTES

\section{CReCIMIENTO EN EL NÚMERO DE HOMICIDIOS POR ESTAdO ENTRE 2007 Y 2017}

En la Tabla 5 es posible observar el total de homicidios ocurridos y su crecimiento en 2007 y 2017 por entidad federativa. Aunque se pudo haber utilizado tasas para relativizar el número de homicidios, ello habría dado como resultado conocer la frecuencia con la que ocurre este fenómeno respecto al total de la población de la entidad. Consideramos que ello escondería la magnitud de los homicidios y la fuerza de su crecimiento por entidad federativa, y en este trabajo nos interesa dar visibilidad a estos aspectos.

El indicador de crecimiento mide la razón de los homicidios de 2017 respecto a los homicidios del 2007. En nuestro caso, los homicidios por estado en 2017 respecto a los de 2007 siempre son más elevados, es decir, en todos los estados ha crecido el número de homicidios.

Las seis entidades federativas en donde los homicidios crecen más lentamente, y por tanto las podríamos calificar como aquéllas entidades federativas donde menos ha escalado el clima de violencia, son: Aguascalientes (los homicidios de 2017 son dos veces mayores a los ocurridos en 2007), Durango (1.1 veces), Yucatán (1.1 veces), Oaxaca (1.8 veces), Ciudad de México (1.6 veces), Sonora (2.0 veces) (ver Tabla 5).

De 2007 y 2017, las 20 entidades federativas donde más ha crecido la violencia del país son: Colima (los homicidios en 2017 son 20.2 veces los de 2007), Baja California Sur (21.5 veces), Guanajuato (10.5 veces), Zacatecas ( 9.5 veces), Tamaulipas ( 6.2 veces), Baja California (5.9 veces), Chiapas (5.9 veces), Morelos (5.1 veces), Veracruz ( 4.9 veces), Nayarit (4.4 veces), Chihuahua (4.3 veces), Hidalgo (4.2 veces), Sinaloa (4.1 veces), Puebla ( 4.0 veces), Querétaro (4.0 veces), Quintana Roo (3.8 veces), Jalisco ( 3.6 veces), San Luis Potosí (3.5 veces), Tlaxcala (3.4 veces), Guerrero (3.4 veces) (ver Tabla 5).

Finalmente, hay seis entidades federativas que experimentaron una escalada de violencia intermedia, ya que los homicidios crecieron de 2.3 a tres veces. Esas entidades federativas son: Campeche ( 2.6 veces), Coahuila (2.5 veces), México (2.5 veces), Michoacán (3.1 veces), Nuevo León (2.4 veces), Tabasco (3.1 veces), (ver Tabla 5).

Como se puede apreciar, ninguna de las entidades federativas presenta una reducción en el número de homicidios (ver Tabla 5). Únicamente en Yucatán y Durango el número de homicidios se incrementó muy levemente (1.1 veces cada uno). 
Tabla 5: Estados Unidos Mexicanos: Número de homicidios por estado en 2007 y 2017 y crecimiento en el periodo 2007 - 2017

\begin{tabular}{|c|c|c|c|}
\hline Estados & 2007 & $2017^{*}$ & $\begin{array}{r}\text { Crecimiento** } \\
2007-2017 \\
\end{array}$ \\
\hline Aguascalientes & 42 & 84 & 2.0 \\
\hline Baja California & 369 & 2,169 & 5.9 \\
\hline Baja California Sur & 35 & 751 & 21.5 \\
\hline Campeche & 50 & 78 & 2.6 \\
\hline Coahuila & 111 & 275 & 2.5 \\
\hline Colima & 43 & 867 & 20.2 \\
\hline Chiapas & 101 & 591 & 5.9 \\
\hline Chihuahua & 518 & 2,248 & 4.3 \\
\hline Ciudad de México & 847 & 1,320 & 1.6 \\
\hline Durango & 174 & 200 & 1.1 \\
\hline Guanajuato & 219 & 2,285 & 10.5 \\
\hline Guerrero & 766 & 2,637 & 3.4 \\
\hline Hidalgo & 74 & 314 & 4.2 \\
\hline Jalisco & 445 & 1,586 & 3.6 \\
\hline México & 1,238 & 3,076 & 2.5 \\
\hline Michoacán & 563 & 1,732 & 3.1 \\
\hline Morelos & 128 & 654 & 5.1 \\
\hline Nayarit & 108 & 474 & 4.4 \\
\hline Nuevo León & 279 & 664 & 2.4 \\
\hline Oaxaca & 564 & 1,018 & 1.8 \\
\hline Puebla & 273 & 1,083 & 4.0 \\
\hline Querétaro & 55 & 218 & 4.0 \\
\hline Quintana Roo & 121 & 455 & 3.8 \\
\hline San Luis Potosí & 143 & 507 & 3.5 \\
\hline Sinaloa & 398 & 1,640 & 4.1 \\
\hline Sonora & 323 & 761 & 2.4 \\
\hline Tabasco & 146 & 450 & 3.1 \\
\hline Tamaulipas & 193 & 1,204 & 6.2 \\
\hline Tlaxcala & 37 & 126 & 3.4 \\
\hline Veracruz & 380 & 1,851 & 4.9 \\
\hline Yucatán & 49 & 52 & 1.1 \\
\hline Zacatecas & 75 & 709 & 9.5 \\
\hline Total & 8,867 & 32,079 & 3.6 \\
\hline
\end{tabular}


El resto de las entidades federativas tuvieron incrementos que van de 1.5 veces en la Ciudad de México a 21.5 veces en Baja California Sur y a 20.2 veces en Colima.

El incremento de los homicidios entre 2007 y 2017 fue de 23,212, 61 por ciento de esta cifra se presentó en los estados de México, Baja California, Chihuahua, Veracruz, Michoacán, Jalisco, Sinaloa, Tamaulipas, Colima, Puebla, Zacatecas y Oaxaca. En estos estados es donde más se ha concentrado la reproducción del clima de violencia.

El comportamiento diferencial en el incremento de los homicidios ha hecho que de 2007 a 2017 las entidades federativas avancen o retrocedan en el lugar que ocupan a nivel nacional por sus niveles de violencia. En las tablas 6 y 7 podemos apreciar que entre 2007 y 2017 ganan lugares Guerrero (pasa del lugar tres al dos), Chihuahua (pasa del seis al cuatro) y Baja California (pasa del diez al cinco), lo que quiere decir que se vuelven más violentos. Se incorpora Guanajuato dentro de los primeros diez en 2017, el cual pasa del lugar catorce al tres. El Estado de México en valor absoluto es el que tiene más homicidios y mantiene el primer lugar en el periodo analizado.

Entre el lugar 11 y el 20 ganan lugares en este mismo periodo Tamaulipas (pasa del 15 al 11), Puebla pasa del lugar 13 al 12. Morelos (se mantiene en el lugar 19). Destaca Colima (pasa del 29 al 14) que multiplicó 20 veces el número de muertos por homicidios en el periodo (Tabla 5).

Pierden lugares, es decir, el incremento de los homicidios fue considerablemente menor en Sonora (pasa del 11 al 15), Nuevo León (pasa del 12 al 18), Tabasco (pasa del 17 al 24), Durango (pasa del 16 al 28) y San Luis Potosí (pasa del 18 al 21).

Entre el lugar 21 y el 32 ganan lugares o se mantienen, Nayarit (se mantiene en el lugar 22), Hidalgo (se mantiene en el lugar 25), Aguascalientes (se mantiene en el lugar 30), Baja California Sur (pasa del 32 al 16), Chiapas (pasa del 23 al 20). Pierden lugares, es decir, mejoran, Coahuila (pasa del 21 al 26), Querétaro (pasa del 26 al 27), Campeche (pasa del 27 al 31) y Yucatán (pasa del 28 al 32).

Entre 2007 y 2017 lo más sobresaliente que se observa en la Tabla 7 es que el estado de Guanajuato pasa al tercer lugar. Chiapas, Zacatecas, Colima y Baja California Sur pasan del grupo de los últimos doce al grupo de los primeros veinte. Analizando las Tablas 6 y 7 se observa que de 2007 a 2017 los homicidios se incrementaron de 8,867 a 32,079, es decir, aumentaron 3.6 veces, y de 2016 a 2017 el número de homicidios aumentó en 30 por ciento, cifras que representan un incremento inmenso. 
Tabla 6: Estados Unidos Mexicanos: Orden de los estados de acuerdo con el número de homicidios, 2007

\begin{tabular}{|c|c|c|c|c|c|}
\hline Lugar & Estado & Total & Hombres & Mujeres & No especificados \\
\hline & País & 8,867 & 7,776 & 1,083 & 8 \\
\hline \multicolumn{6}{|c|}{ Los primeros 10} \\
\hline $1^{\circ}$ & México & 1,238 & 1,049 & 188 & 1 \\
\hline $2^{\circ}$ & Ciudad de México & 847 & 725 & 122 & 0 \\
\hline $3^{\circ}$ & Guerrero & 766 & 686 & 80 & 0 \\
\hline $4^{\circ}$ & Oaxaca & 564 & 493 & 71 & 0 \\
\hline $5^{\circ}$ & Michoacán & 563 & 513 & 50 & 0 \\
\hline $6^{\circ}$ & Chihuahua & 518 & 460 & 53 & 5 \\
\hline $7^{\circ}$ & Jalisco & 445 & 390 & 55 & 0 \\
\hline $8^{\circ}$ & Sinaloa & 398 & 369 & 29 & 0 \\
\hline $9^{\circ}$ & Veracruz & 380 & 334 & 46 & 0 \\
\hline $10^{\circ}$ & Baja California & 369 & 343 & 26 & 0 \\
\hline \multicolumn{6}{|c|}{ Del 11 al 20} \\
\hline $11^{\circ}$ & Sonora & 323 & 296 & 27 & 0 \\
\hline $12^{\circ}$ & Nuevo león & 279 & 256 & 23 & 0 \\
\hline $13^{\circ}$ & Puebla & 273 & 229 & 44 & 0 \\
\hline $14^{\circ}$ & Guanajuato & 219 & 188 & 31 & 0 \\
\hline $15^{\circ}$ & Tamaulipas & 193 & 165 & 26 & 2 \\
\hline $16^{\circ}$ & Durango & 174 & 165 & 9 & 0 \\
\hline $17^{\circ}$ & Tabasco & 146 & 127 & 19 & 0 \\
\hline $18^{\circ}$ & San Luis Potosí & 143 & 119 & 24 & 0 \\
\hline $19^{\circ}$ & Morelos & 128 & 110 & 18 & 0 \\
\hline $20^{\circ}$ & Quintana Roo & 121 & 101 & 20 & 0 \\
\hline \multicolumn{6}{|c|}{ Del 21 al 32} \\
\hline $21^{\circ}$ & Coahuila & 111 & 97 & 14 & 0 \\
\hline $22^{\circ}$ & Nayarit & 108 & 97 & 11 & 0 \\
\hline $23^{\circ}$ & Chiapas & 101 & 88 & 13 & 0 \\
\hline $24^{\circ}$ & Zacatecas & 75 & 64 & 11 & 0 \\
\hline $25^{\circ}$ & Hidalgo & 74 & 62 & 12 & 0 \\
\hline $26^{\circ}$ & Querétaro & 55 & 47 & 8 & 0 \\
\hline $27^{\circ}$ & Campeche & 50 & 43 & 7 & 0 \\
\hline $28^{\circ}$ & Yucatán & 49 & 36 & 13 & 0 \\
\hline $29^{\circ}$ & Colima & 43 & 37 & 6 & 0 \\
\hline $30^{\circ}$ & Aguascalientes & 42 & 35 & 7 & 0 \\
\hline $31^{\circ}$ & Tlaxcala & 37 & 25 & 12 & 0 \\
\hline $32^{\circ}$ & Baja California Sur & 35 & 27 & 8 & 0 \\
\hline
\end{tabular}

Fuente: INEGI. 
El fin de la esperanza: los homicidios como causa de la expectativa de vida perdida / M. ORDORICA y M.P. CERVANTES

Tabla 7: Estados Unidos Mexicanos: Orden de los estados de acuerdo con el número absoluto de homicidios, 2017

\begin{tabular}{|c|c|c|c|c|c|}
\hline Lugar & Estado & Total & Hombres & Mujeres & No especificados \\
\hline & País & 31,174 & 27,771 & 3,324 & 79 \\
\hline \multicolumn{6}{|c|}{ Los primeros 10} \\
\hline $1^{\circ}$ & México & 3,046 & 2,574 & 462 & 10 \\
\hline $2^{\circ}$ & Guerrero & 2,578 & 2,357 & 214 & 7 \\
\hline $3^{\circ}$ & Guanajuato & 2,252 & 2,037 & 215 & \\
\hline $4^{\circ}$ & Chihuahua & 2,221 & 1,965 & 256 & \\
\hline $5^{\circ}$ & Baja California & 2,144 & 1,933 & 202 & 9 \\
\hline $6^{\circ}$ & Michoacán & 1,661 & 1,517 & 142 & 2 \\
\hline $7^{\circ}$ & Sinaloa & 1,617 & 1,532 & 83 & 2 \\
\hline $8^{\circ}$ & Jalisco & 1,582 & 1,424 & 142 & 16 \\
\hline $9^{\circ}$ & Veracruz & 1,555 & 1,376 & 175 & 4 \\
\hline $10^{\circ}$ & Ciudad de México & 1,315 & 1,174 & 140 & 1 \\
\hline \multicolumn{6}{|c|}{ Del 11 al 20} \\
\hline $11^{\circ}$ & Tamaulipas & 1,174 & 1,023 & 139 & 12 \\
\hline $12^{\circ}$ & Puebla & 1,070 & 929 & 141 & \\
\hline $13^{\circ}$ & Oaxaca & 858 & 740 & 117 & 1 \\
\hline $14^{\circ}$ & Colima & 848 & 769 & 75 & 4 \\
\hline $15^{\circ}$ & Sonora & 752 & 684 & 63 & 5 \\
\hline $16^{\circ}$ & Baja California Sur & 740 & 662 & 77 & 1 \\
\hline $17^{\circ}$ & Zacatecas & 691 & 599 & 92 & \\
\hline $18^{\circ}$ & Nuevo León & 663 & 601 & 62 & \\
\hline $19^{\circ}$ & Morelos & 626 & 557 & 69 & \\
\hline $20^{\circ}$ & Chiapas & 593 & 526 & 67 & \\
\hline \multicolumn{6}{|c|}{ Del 21 al 32} \\
\hline $21^{\circ}$ & San Luis Potosí & 500 & 451 & 48 & 1 \\
\hline $22^{\circ}$ & Nayarit & 462 & 405 & 57 & \\
\hline $23^{\circ}$ & Quintana Roo & 450 & 402 & 47 & 1 \\
\hline $24^{\circ}$ & Tabasco & 438 & 393 & 45 & \\
\hline $25^{\circ}$ & Hidalgo & 317 & 265 & 49 & 3 \\
\hline $26^{\circ}$ & Coahuila & 273 & 232 & 41 & \\
\hline $27^{\circ}$ & Querétaro & 217 & 182 & 35 & \\
\hline $28^{\circ}$ & Durango & 197 & 170 & 27 & \\
\hline $29^{\circ}$ & Tlaxcala & 125 & 109 & 16 & \\
\hline $30^{\circ}$ & Aguascalientes & 83 & 75 & 8 & \\
\hline $31^{\circ}$ & Campeche & 76 & 70 & 6 & \\
\hline $32^{\circ}$ & Yucatán & 50 & 38 & 12 & \\
\hline
\end{tabular}

Fuente: INEGI, cifras preliminares de 2017 que difieren en 905 homicidios respecto a las cifras definitivas, pero que no cambian el orden por estado en el número de homicidios.

Estos resultados nos invitan a preguntarnos sobre las estrategias que han seguido los gobiernos donde la violencia se ha reducido, y la pertinencia de su adaptación a contextos en los que la violencia se está incrementado. 
La cifra preliminar publicada por el INEGI sobre los homicidios para 2018, con corte al 15 de julio de 2019, fue de 35, 964, lo que equivale a una tasa de 26 homicidios por cada 100 mil habitantes, tasa superior a la de 2017 que fue de 25.86 por 100 mil. Es importante destacar que, según estos datos, en 2018 fallecieron por homicidio 32,141 hombres por 3,663 mujeres, lo que da una razón de 8.77 hombres por cada mujer. Según estos mismos datos, del total de muertes por homicidio, 24,349 fueron por agresión con arma de fuego, lo que representa 67.7 por ciento del total. Es importante señalar que estas últimas cifras no se han incorporado en las tablas del texto con excepción de la Figura 5, debido a que, como dice la nota del INEGI: "aún no concluyen los procesos de generación de la estadística de muertes registradas."

A nivel de estado se encuentran resultados importantes en el incremento de los homicidios entre 2017 y 2018 (ver Figura 5). Sólo por presentar algunos de los principales cambios se observa que en Baja California en este periodo el número de homicidios aumentó de 2,169 a 2,910 (1.3 veces); en Chihuahua pasó de 2,248 a 2,957 (1.3 veces), en Guanajuato cambió de 2,285 a 3,493 (1.5 veces) y en Jalisco el incremento fue de 1,586 a 2,919 (1.8 veces). ${ }^{8}$

En la cifra acumulada de 2009 a 2018 se observa en la Figura 5 que Chihuahua tiene el primer lugar en homicidios; Guerrero, el segundo lugar; Sinaloa, el tercer lugar; Colima, el cuarto y Baja California el quinto lugar. Llama la atención en la Figura 5 como el estado de Colima cuenta con la tasa por homicidios más elevada en 2018.

En la misma Figura 5 se observa que las entidades federativas que cuentan con las tasas acumuladas más bajas sobre homicidios en el periodo 2009-2018 son: Yucatán, con el nivel más bajo, Aguascalientes, Tlaxcala, Hidalgo y Campeche.

\footnotetext{
7 Fuente: INEGI. Estadísticas Vitales. Defunciones registradas. Cifras preliminares con corte al 15 de julio de 2019. https://www.inegi.org.mx/programas/mortalidad/

${ }^{8}$ Fuente: op. cit. INEGI. Estadísticas Vitales Defunciones Registradas. Cifras preliminares con corte al 15 de julio de 2019. https://www.inegi.org.mx/programas/mortalidad/
} 
Figura 5: Homicidios por cada 100,000 habitantes por estado, 2009-2018

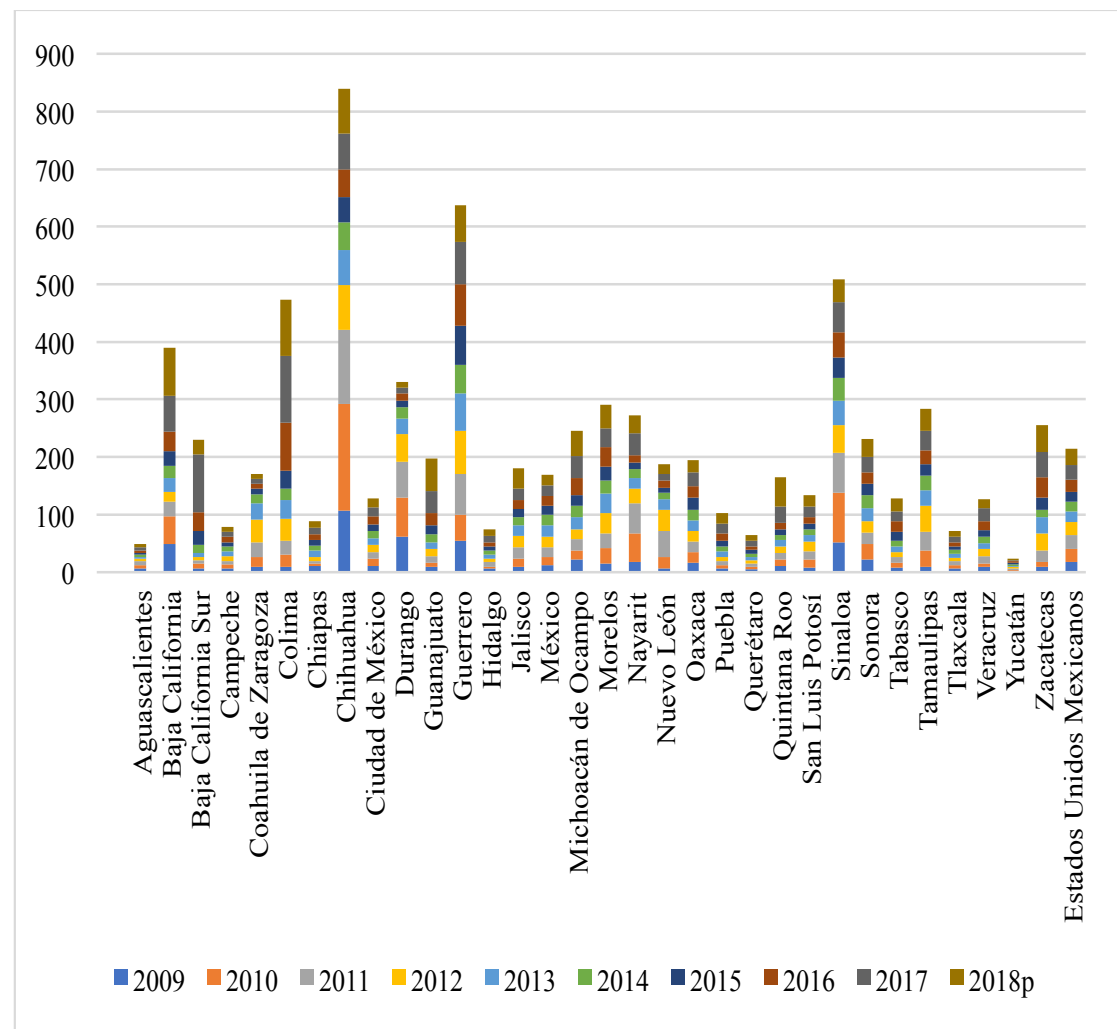

Fuente: INEGI. Estadísticas Vitales. Defunciones registradas.

p. Cifras preliminares con corte al 15 de julio de 2019.

\section{Conclusiones}

El comportamiento de los homicidios a lo largo de la historia nos permite señalar que la época que vivimos no es la única violenta en la historia de México, ya que los años cuarenta del siglo XX fueron momentos de altos niveles de mortalidad por homicidios, y en la actualidad estamos regresando a una situación similar.

Aunque el clima de violencia no es inédito, el número de homicidios sí lo es; en el periodo de 35 años, de 1982 a 2017, ocurrieron 500 mil homicidios. Sólo en 11 años, entre 2006 y 2017, el número de fallecimientos por homicidios fue de 251,932 personas. En Francia durante la Segunda Guerra Mundial murieron alrededor de 250 mil soldados (Van, 1978). 
El Instituto Internacional de Estudios Estratégicos, insiste en señalar que México se encuentra en un conflicto armado, entendido como una "lucha sostenida entre dos o más adversarios organizados, haciendo uso intencional de la fuerza armada. De manera crucial, involucra el combate, en lugar de la aplicación unilateral de la fuerza letal." En este caso hay diversos grupos del narco y otros grupos violentos en pugna y el Estado Mexicano, en donde muchas de las victimas salen de la población civil (IIES, 2017).

En cuanto al impacto demográfico de la violencia, hemos de decir que nuestro país va a pasar a la historia, ya que la esperanza de vida al nacer ya no ha aumentado en la última década, nos estancamos en 75 años. Hoy ya deberíamos de tener unos cuatro años más en la esperanza de vida al nacer si hubiéramos seguido la trayectoria de Japón cuando llegó a los 75 años.

En cuanto a la evolución de la violencia en los territorios del país, destacan los siguientes resultados. De 2007 a 2017 el estado de México presenta los números absolutos más elevados y con ello ha ocupado el primer lugar en el número de muertes por homicidios. Por su parte, Colima y Baja California Sur presentaron el crecimiento más rápido en el número de homicidios. Casi las dos terceras partes de los homicidios en el país en 2016 y 2017 ocurrieron en diez entidades federativas: Estado de México, Guerrero, Chihuahua, Michoacán, Jalisco, Sinaloa, Ciudad de México, Guanajuato, Baja California y Veracruz. Debe reconocerse que la violencia es un fenómeno extendido a lo largo del territorio mexicano, y que el inicio del siglo XXI es uno de los momentos más violentos de nuestra historia mexicana. En contra parte a este clima de violencia generalizado, destacan Campeche y Yucatán, entidades federativas que en el año 2017 sobresalieron con el menor número de muertes violentas.

El análisis por edad y sexo de las muertes por homicidios, mostraron que casi se mueren ocho veces más hombres que mujeres por homicidios, y el máximo en el número de homicidios por edad se ubica en el grupo 25 a 29 años, aunque el número de homicidios en los grupos de edades vecinos es casi tan elevado como el valor máximo. Las implicaciones demográficas de ello es que se están perdiendo los elementos que forman parte del bono demográfico, sobre todo los hombres.

La información de registros administrativos referentes a la mortalidad por homicidios que tenemos hoy, si bien es cierto que son de buena calidad, se publican con retraso, por lo que no pueden ser vistos como un instrumento que sirva para la reacción inmediata. No obstante, son datos confiables y de gran relevancia para el diseño de política pública con el fin de intentar superar el grave problema de las muertes violentas en México 
y sus territorios. Estos resultados nos permiten avanzar las siguientes preguntas: ¿Cuáles son las condiciones sociales, económicas y políticas que han favorecido la reproducción diferencial de la violencia en los territorios del país? ¿Tendría que existir una estrategia generalizada o diferenciada según el comportamiento particular de la violencia y sus causas? Es decir, y retomando la propuesta relacional entre la violencia estructural, cultural y directa de Galtung (2016: 154), ¿Cuáles son las fuentes estructurales y culturales que explican la violencia directa y su comportamiento diferencial entre entidades federativas?

Los datos ofrecidos a lo largo de este documento muestran que falta mucho por hacer para garantizar el derecho a la vida y la seguridad de las personas en México. El Estado Nacional y los gobiernos estatales no han logrado garantizar este derecho, y el estancamiento en la esperanza de vida y el incremento en las muertes por homicidios, dan muestra de la importante erosión que ha provocado el clima de violencia en el derecho a la vida.

\section{REFERENCIAS BIBLIOGRÁFICAS}

Aguirre, A., Vela, F. y Gallegos A., abril 2015, "Years of life lost in the war against drug cartels in México during the government of Calderón, 2006-2012”. Poster presentado en la sesión P5 'Adult Health and Mortality', en la Reunión Anual de la Population Association of America, San Diego, California.

Bravo Becherelle, M y Reyes Jiménez, R., 1958, "Tablas de vida para México, 1895 a 1956", en Revista del Instituto de Salubridad y Enfermedades Tropicales, vol. 18, núm. 2, México, D.F.

Calderón C. P., 2009, “Teoría de conflictos de Johan Galtung”, en Revista de Paz y Conflictos, núm. 2, pp. 60-81

Camposortega C. S., 1992, Análisis Demográfico de la Mortalidad en México, 1940-1980. México: El Colegio de México.

Camposortega, C. S., 1989, "La evolución de la mortalidad en México", 19401980, en Estudios Demográficos y Urbanos, (4) 2, pp. 229-264.

Canudas-Romo, V., Echarri, C. y García V., 2014, "The stagnation of the mexican male life expectancy in the first decade of the 21st. Century: The impact of homicides and diabetes mellitus", in J. Epidemmiol Community Health. Online first: 24 September 2014. DOI: 10-1136/jech-2014-204237.

Cárdenas, R., 2014, "Mortalidad: niveles, cambios y necesidades en materia de política pública", en Rabell, C., (coord.). Los mexicanos. Un balance del cambio demográfico (pp. 115-133). México: Fondo de Cultura Económica.

Cendejas, G. J. y Merino, P. L., 2016, “Acción colectiva en la construcción social de la paz y la seguridad. La paz y la seguridad como bienes comunes", en Cultura y Representaciones Sociales, 10 (20), pp. 9-41. 
Chaparro-Narváez, P., Cotes-Cantillo, K., León-Quevedo, W., Castañeda-Orjuela, C., 2016, "Mortalidad por homicidios en Colombia, 1998-2012", en Biomédica, (36)4, pp. 572-582.

CONAPO, 2017, Estimaciones y proyecciones de la población de México por entidad federativa, 2010-2050. México: Proyecciones de la población 2010-2050. Consejo Nacional de Población Recuperado de http://www.conapo.cob.mx

CONAPO, 2002, Proyecciones de la población de México, 2000-2050. México: Consejo Nacional de Población (CONAPO).

Escalante, F., 2009, El homicidio en México entre 1990 y 2007. Aproximación estadística. México: COLMEX, CEI y SSPF.

Escalante, F., 2015, El crimen como realidad y representación. México: El Colegio de México.

Galtung, J., 2016, "La violencia cultural, estructural y directa", en Cuadernos de estrategia, núm. 183, pp. 147-168.

Hiik, 2018, Conflict Barometer. No. 26. Heidelberg: Heidelberg Institute for International Conflict Research (HIIK). Disponible en https://www.iiss.org/blogs/ analysis/2018/07/revisions-and-review

INEGI, 2015, Registros Administrativos, defunciones por homicidio, 2000-2010. México: Instituto Nacional de Estadística y Geografía (INEGI). Consulta interactiva de datos.

INEGI, 2019, Estadísticas Vitales. Defunciones Registradas. Cifras preliminares con corte al 15 de julio de 2019. Instituto Nacional de Estadística y Geografía (INEGI). Disponible en https://www.inegi.org.mx/programas/mortalidad/

IIES, 2017, Armed Conflict Survey 2017. United States: International Institute for Estrategy Studies (IIES). Disponible en https://www.iiss.org/blogs/analysis/2018/07/revisions-and-review

Lotka, Alfred J., 1969, Teoría Analítica de las Asociaciones Biológicas. Centro Latinoamericano de Demografía (CELADE). Santiago de Chile.

Naciones Unidas, s/f, Declaración Universal de Derechos Humanos. Disponible en http://www.un.org/es/universal-declaration-human-rights/

Ordorica, M., 2015, "En Ciudad Juárez los hombres se mueren también y su esperanza de vida se reduce", en Coyuntura Demográfica, 9, pp. 15-21.

OMS, 2003, Informe mundial sobre la violencia y la salud. Washington: Organización Panamericana de la Salud, Oficina Regional para las Américas de la Organización Mundial de la Salud (OMS).

PAHO, s/f, México, tasas de homicidios por 100 mil habitantes desde 1931 a 2016. Recopilación del Ingeniero Manuel Aguirre Botello, con datos de INEGI, SINAIS, Panamerican Health Organization (PAHO), Presidencia y Lantia.

Paz, O., 1999, El laberinto de la Soledad. México: FCE. 
Rosquillo, V., 1999, Las muertas de Juárez, Crónica de una larga pesadilla. México: Planeta.

Salama, P., 2013, "Homicidios, jes ineluctable la violencia en América?", en Frontera Norte, 25 (49), pp.7-27.

Silva E., Guerrero V.M y Peña D., 2014, "Suavizamiento controlado de las tasas de mortalidad con P-Splines: aplicaciones para México y el Reino Unido", en Papeles de Población, vol. 20, núm. 79, pp. 99-131

United Nations, 2017, World Population Prospects. Departament of Economic and Social Affairs, Population Division. The 2017 Revision. New York.

Van M. W., 1978, Bilanz des Krieges. Rotterdam: Lekturama.

Victoria, L. M., Hijar, M. M., Rascón P. R. y Blanco M. J., 1996, “Muertes por homicidio, consecuencia fatal de la violencia. El caso de México, 1979-1992”, en Saúde Pública, 30 (1), pp. 46-52.

World Bank, 2018, Life expectancy at bird. Data World Bank. Disponible en https://data.worldbank.org/indicator/SP.DYN.LE00.IN?locations=JP

\section{RESUMEN CURRICULAR DE LOS AUTORES}

\section{Manuel Ordorica Mellado}

Actuario de la Facultad de Ciencias de la UNAM; maestro en demografía de El Colegio de México y doctor en Investigación de Operaciones en la UNAM. Realizó una especialidad en Análisis Demográfico en la Agencia de Cooperación Internacional de Japón. Fue jefe de Evaluación Demográfica en la Dirección General de Estadística y director de Estudios de Población en el CONAPO; consultor en Educación en Población en la UNESCO. Coordinó la Maestría en Demografía y el Doctorado en Estudios de Población en El Colegio de México. Fue Director y Secretario General en el mismo Colegio. Formó parte del Consejo Editorial de la revista Population del INED en París. Fue miembro del Comité técnico para la Redistritación y para la Evaluación del Padrón Electoral en el INE. Pertenece al Sistema Nacional de Investigadores nivel III. En 1998 recibió el Premio Nacional de Demografía. Realizó una estancia sabática en el CentroGeo de Conacyt. Actualmente es profesor-investigador de El Colegio de México y trabaja en demografía matemática. En 2015 se publicó en la revista Coyuntura Demográfica el artículo: En Ciudad Juárez los hombres se mueren también y su esperanza de vida se reduce. En la misma revista, en 2018, se publicó el artículo: Estimaciones de la población de áreas pequeñas desde la Tierra como del Cielo. El caso de Tenosique. En 2016 se publicó su libro sobre los Grandes Problemas: Una mirada al futuro demográfico de México, editado por El Colegio de México y la UNAM. En 2017 Springer 
publicó el artículo: Estimación de la población de áreas pequeñas a través del Filtro de Kalman.

Dirección electrónica: mordori@colmex.mx

Registro ORCID: https://orcid.org/0000-0002-7649-1729

\section{Mauricio Pablo Cervantes Salas}

Licenciado en Biología por la Facultad de Ciencias de la Universidad Nacional Autónoma de México; maestro en Demografía y doctor en Estudios de Población por el Colegio de México A.C. Actualmente es profesor investigador del Centro de Investigación en Ciencias de Información Geoespacial A.C. (CentroGeo), institución en la que ha impartido el seminario "Manejo comunitario de recursos naturales" para la maestría en geomática, y el curso "Espacio y Desigualdad" para la maestría en Planeación Espacial. Fue Jefe de Departamento de Desarrollo Sustentable en la Dirección de Poblamiento y Desarrollo Regional Sustentable, de la Secretaría General del Consejo Nacional de Población. Sus temas de interés en la investigación están relacionados con desigualdad, familia, territorio y manejo comunitario de recursos naturales. En 2020 publicó como primer autor en la revista Estudios Demográficos y Urbanos el artículo: Territorialización del capital social: apropiación forestal comunitaria en la frontera entre Tabasco y Chiapas. En 2021 escribió el capítulo del libro: Dinámicas territoriales en la zona del Soconusco guatemalteco, publicado en el libro Entre lo político y lo espacial: representaciones geopoliticas de la región transfrotenriza México-Guatemala, editado por CentroGeo. En el mismo libro, escribió como segundo autor, el capítulo titulado: Comprendiendo las redes de apoyo laboral de los migrantes circulares guatemaltecos: análisis del espacio trasnacional del mercado laboral transfroterizo.

Dirección electrónica: mcervantes@centrogeo.edu.mx

Registro ORCID: https://orcid.org/0000-0002-0173-2735 